A myriad tiny magnets in fluid, trapped inside a glass ball, swirl and convect in a magnetic field mirroring the earth's liquid mantle

\title{
Magnetic Fluids: Modelling the Earth's Mantle in a Glass Ball
}

\section{Ronald E. Rosensweig}

$T^{n}$ the earth's liquid mantle, thermal $I$ convection drives contifnental drift, recreating the shape of the ocean floor (as first made convincing by Alfred Wegener in 1912). In fact thermal convection is the major source of movement in the mantle, coupling the changing surface of the earth to the interior. The cross sectional sketch of the earth shown in figure 1 illustrates this. Because the gravitational field must be central it has previously not been possible to study mantle convection in a terrestrial laboratory. Experiments have been conducted on the space shuttle: convection in a rotating shell of fluid using the

fluid using
the

weak but central force field of an electrostatic source. But magnetic fluids offer an alternative, and the means to establish central body-force fields in the laboratory that greatly exceed the force of gravity.

Magnetic fluids (also called ferrofluids) are colloidal dispersions of tiny magnetic particles (typically $10 \mathrm{~nm}$ across) in liquid carriers stabilized with one or two layers of surfactants or electrical charges on their surface. The small size of the particles gives thermal motion that prevents the particles from settling out in gravitational or magnetic fields, while the sorbed layers serve as a cushion to prevent particles from sticking together when they collide. The fluids have been studied because of their novel behaviour (such as the sudden appearance of liquid peaks in a patterned array, that form on the surface of a pool of ferrofluid when a magnetic field exceeds a critical density) while a number of applications have found their way into the marketplace (such as zero leakage rotary seals for semiconductor manufacture, exclusion seals found in nearly all computer hard disk drives, sink/float mineral separation processes, low run out hydrodynamic bearings used in laser printers to rotate scanning mirrors, and dampers for precision machinery such as stepper motors). The property of a magnetic fluid to retain its liquid flowability in the highest applied magnetic fields is key to many of its uses.

In the absence of a magnetic field, the magnetic dipolar particles in the fluid are oriented randomly due to thermal motion, and the bulk fluid is unmagnetized. In an external magnetic field $\mathbf{H}$ the particles tend to align and a net magnetization $M$ develops. When the field is spatially non-uniform a force density $g_{0}=\mu_{0} M \mid \nabla$ $\mathbf{H} \mid / \rho$ acts on the fluid in the direction of increasing field where $M$ and $H$ are magnitudes of the vectors, $\rho$ is mass density, and $\mu_{0}$ is the permeability of free space. Magnetization is temperature dependent, decreasing with an increase in temperature. In the presence of a temperature gradient in space, the resulting variation in $M$ can induce fluid motion (analogous to density variation in buoyant convection) with cooler fluid drawn into regions of higher magnetic field and warmer fluid displaced to regions of lower field intensity. Thus, if $\nabla T$ is parallel to $\nabla H$ a situation of thermal instability can develop-similar to the Rayleigh-Benard phenomenon in ordinary fluids where heated fluid is buoyant in a gravity field and is set into circulatory motion. This situation corresponds to the thermal conditions across the earth's mantle.

Due to cold flow creep, a process in which ions and vacancies diffuse through the crystal lattice, over geologic time periods the mantle rock is believed to flow like a Newtonian fluid (for which strain is directly proportional to stress). Analysis of the governing differential equations of fluid motion and energy conservation show that flow and temperature distribution are determined by the sum of the ordinary Rayleigh number $R a$ and the magnetic Rayleigh number $R a_{\mathrm{m}}$.

$$
\mathbf{R a}=\frac{g \alpha \Delta \mathbf{T d}^{3}}{\kappa v} \quad \mathrm{Ra}_{\mathrm{m}}=\frac{\mathrm{g}_{0} \beta \Delta \mathrm{Td}^{3}}{\kappa v}
$$

where $\alpha=-(1 / \rho) \partial \rho / \partial T$ is the coefficient of thermal expansion $\beta=-(1 / M) \partial M / \partial T$, $\Delta T$ is the temperature difference, $d$ the layer thickness, $k$ the thermal diffusivity, and $v$ the kinematic viscosity. For earth the magnetic Rayleigh number is zero while in a suitably designed model $R a_{m}$ is very much greater than $R a$ so that the ordinary Rayleigh number can be neglected. Thus, the similarity of earth and the 

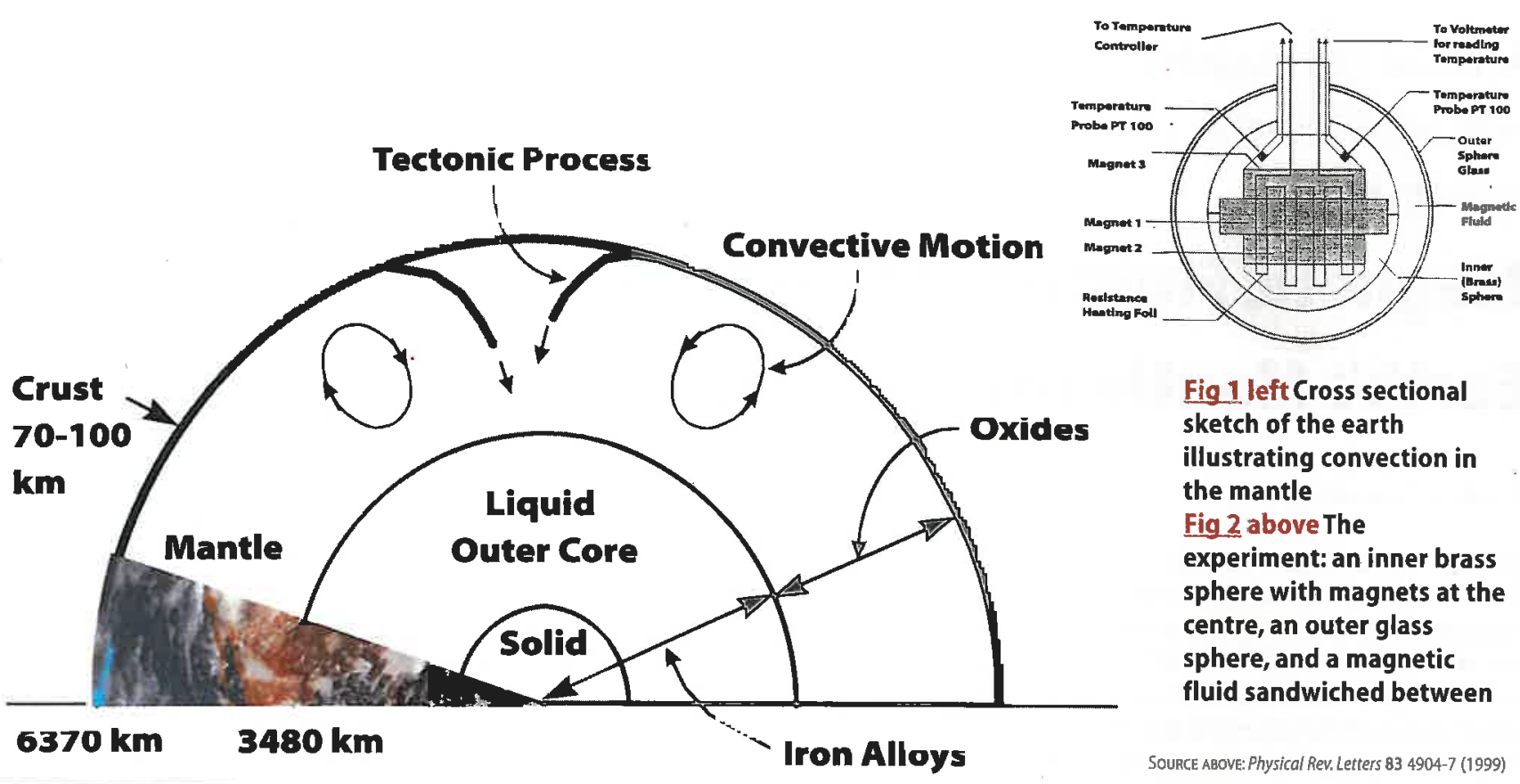

Fig 1 left Cross sectional sketch of the earth illustrating convection in the mantle

Fig 2 above The experiment: an inner brass sphere with magnets at the centre, an outer glass sphere, and a magnetic fluid sandwiched between

SOURCE ABOVE: Physical Rev. Letters 83 4904-7 (1999)

laboratory model requires that $(R a)_{E}=$ $(\text { Ram })_{M}$ where subscript $E$ denotes earth and $M$ the model.

Stability analysis predicts that the heat transfer process is purely conductive until a critical value of Rayleigh number of the order of $10^{3}$ is exceeded. It is estimated that the earth Rayleigh number is about $10^{6}$ and hence far exceeds the critical value. Although recent advances in numerical computation are permitting calculations in this regime it is prudent to establish experimental support to validate the assumptions and the predicted behaviours.

An experimental system has been fabricated. It consists of concentric spheres with magnetic fluid in between. The outer sphere is glass of 50 millimetres inside diameter and the inner sphere is brass of 35 millimetres diameter. The inner sphere contains an electrical heater, temperature probes, and an assembly of permanent magnets (Nd-Fe-B) that are the source of the magnetic field. A simple permanent magnet is dipolar and so does not provide the desired symmetry of field. To overcome this limitation an assembly of three cylindrical magnets is configured (figure 2) to provide a near field over which the distribution of magnitude is reasonably uniform in all directions at a given radius from the centre. By measuring the field it was calculated that simulated gravity averaged 32 times normal gravity at the inner sphere and 6 at the outer sphere for water-based ferrofluids (glycerol-based ferrofluids produced even stronger fields).
Measuring the temperature field on the spherical outer surface is accomplished with an infrared camera. The technique detects conditions over any desired hemisphere so the surface pattern of convection cells, when they are present, can be seen at a glance.

Figure 3a illustrates an equatorial view of the detected thermal pattern when a nonmagnetic liquid (ie water) is heated in the system. As expected, the warmest fluid $\left(56^{\circ} \mathrm{C}\right.$, deep blue) is found at the top and the coolest fluid at the bottom $\left(47^{\circ} \mathrm{C}\right.$, red). The neck seen at the top of the system is actually a tube which holds the inner sphere in place and carries electrical leads to the centre.

Figure $3 \mathrm{~b}$ is a bottom polar view of the system when filled with a water-based ferrofluid. Warm fluid wells up in a convection cell at the bottom of the system transported by thermal convection in the magnetic field, with cooler fluid descending in the surrounding space. Mean value $R a_{m}=$ $1.7 \times 10^{7}$ exceeds the earth value; operation of the system with the more viscous glycerol-based magnetic fluid yields lower operating values of $R a_{m}$. At one point the convection cell displays lobes (figure $3 \mathrm{c}$ ) and continues to change its morphology. Numerical models of mantle convection at high Rayleigh numbers also show convective planforms that are complex and unsteady. The experimental model offers another useful trait-the compression of the time scale by a huge factor. In theory features of convection that have taken the lifetime of the earth to evolve can be de- veloped in short times (less than an hour) in the laboratory.

This work demonstrates the feasibility of the magnetic fluid technique and the means for observing convection cells. A refined model is planned in which the spherical symmetry of the field magnitude is more closely approached by randomly reorienting a central magnet on a time scale that is shorter than time scales of the flow. In addition, other areas of planetary and geophysical research could use magnetic fluids, eg the study of atmospheric circulation, ocean currents, and convection in a liquid core.

\section{Further reading}

Behavior of the Earth-Continental and Seafloor Mobility by C.J. Allègre (Harvard University Press, Cambridge, Massachusetts, 1988) - Ferrohydrodynamics by R.E. Rosensweig (Dover republication of a work published by Cambridge University Press in $1985,1997)$ - Laboratory Study of Spherical Convection in Simulated Central Gravity by R.E. Rosensweig, J. Browaeys, J-C. Bacri, R. Perzynski and A. Zebib Physical Rev. Letters 83 4904-7 (1999)

The author is currently a science consultant in the United States. The work described here was conducted during a twelve month Pascal appointment awarded by the Foundation of the Ecole Normale Supèrieure in Paris

This is an edited version of an article that first appeared in the bulletin of the Société Française de Physique 

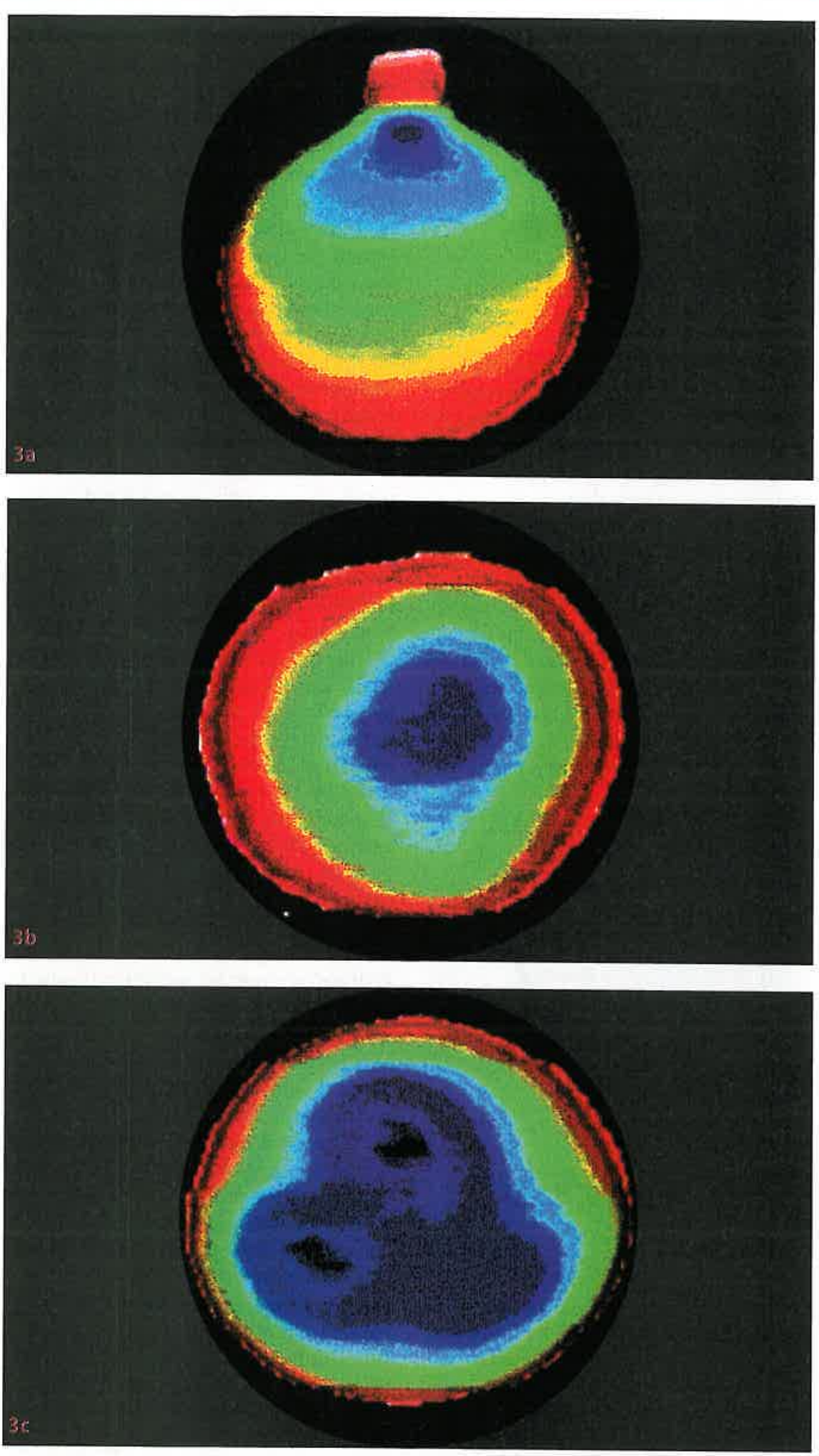

Fig 3 Infrared images colour coded to display temperature distribution at the outer spherical surface of the experiment

a Temperature when operating with nonmagnetic fluid (water). The warmest fluid is at the top, coolest at the bottom. Inner sphere temperature $T_{1}=65^{\circ} \mathrm{C}$

b The warmest fluid is at the bottom due to the convective cell shown in this polar view using aqueous based magnetic fluid in the system $\left(\operatorname{Ra}_{\mathrm{m}}=1.8 \times 10^{7}, \mathrm{~T}_{1}=69^{\circ} \mathrm{C}\right)$ c The convective patterns are time dependent and sensitive to heating conditions; this polar view depicts a three lobed cell (compare with b)

\section{Watch this \\ EUROPHYSICS LETTERS}

\section{Space}

TO COME...

In future issues of europhysics news this column will be reserved for

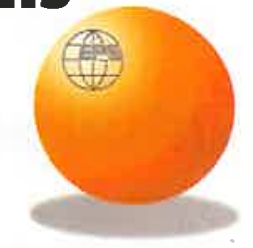

résumés of arti-

EUROPEAN PHYSE AI SOCIETY

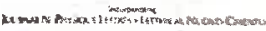
cles that have appeared in Europhysics Letters. Tenth in the world in terms of impact factor, this letters journal was launched more than ten years ago by the European Physical Society, the Société Française de Physique, the Società Italiana di Fisica, and the United Kingdom's Institute of Physics and is now owned by 17 national physical societies and institutes. The journal remains the scientific responsibility of the EPS - this is ensured by carefully selecting 26 co-editors in various areas of physics. Around $\mathbf{3 0}$ advisory editors provide additional expertise and know-how when necessary. EPL receives contributions from all over the world, and is circulated to more than 30 countries. Manuscripts are received at the editorial office in Geneva, and production of the accepted manuscripts is achieved in collaboration with the Societa Italiana di Fisica in Bologna and the publisher EDP Sciences in Paris (also the publisher of europhysics news). Letters submitted to Europhysics Letters are limited to 7 journal pages, must be original and should contain non-trivial new results, ideas, concepts, experimental methods, theoretical treatments etc. and be of broad interest and importance to one or several sections of the physics community. The presentation should satisfy the specialist, yet be understandable to researchers in other fields. There are no page-charges and authors receive $\mathbf{5 0}$ free reprints gratis. For complete information on submission requirements and procedures please vișit the EPL home-

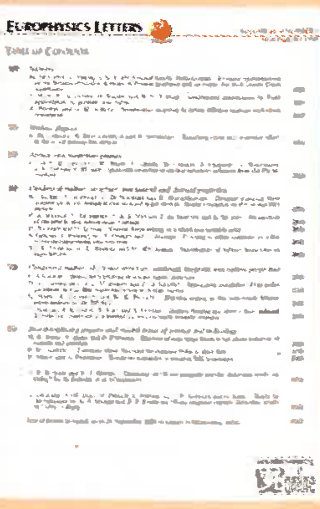
page at www.epletters.ch. 